\title{
Effect of sound level on virtual and free-field localization of brief sounds in the anterior median plane
}

DOI:

10.1016/j.heares.2018.06.004

\section{Document Version}

Accepted author manuscript

Link to publication record in Manchester Research Explorer

\section{Citation for published version (APA):}

Marmel, F., Marrufo-Pérez, M. I., Heeren, J., Ewert, S., \& Lopez-Poveda, E. A. (2018). Effect of sound level on virtual and free-field localization of brief sounds in the anterior median plane. Hearing Research, 365, 28-35. https://doi.org/10.1016/j.heares.2018.06.004

\section{Published in:}

Hearing Research

\section{Citing this paper}

Please note that where the full-text provided on Manchester Research Explorer is the Author Accepted Manuscript or Proof version this may differ from the final Published version. If citing, it is advised that you check and use the publisher's definitive version.

\section{General rights}

Copyright and moral rights for the publications made accessible in the Research Explorer are retained by the authors and/or other copyright owners and it is a condition of accessing publications that users recognise and abide by the legal requirements associated with these rights.

\section{Takedown policy}

If you believe that this document breaches copyright please refer to the University of Manchester's Takedown Procedures [http://man.ac.uk/04Y6Bo] or contact uml.scholarlycommunications@manchester.ac.uk providing relevant details, so we can investigate your claim.

\section{OPEN ACCESS}




\section{Manuscript Details}

\section{Manuscript number}

Title
HEARES_2017_490_R2

Effect of sound level on virtual and free-field localization of brief sounds in the anterior median plane

Research Paper

\section{Article type}

Abstract

The detection of high-frequency spectral notches has been shown to be worse at $70-80 \mathrm{~dB}$ sound pressure level $(\mathrm{SPL})$ than at higher levels up to $100 \mathrm{~dB}$ SPL. The performance improvement at levels higher than $70-80 \mathrm{~dB}$ SPL has been related to an 'ideal observer' comparison of population auditory nerve spike trains to stimuli with and without high-frequency spectral notches. Insofar as vertical localization partly relies on information provided by pinna-based high-frequency spectral notches, we hypothesized that localization would be worse at $70-80 \mathrm{~dB}$ SPL than at higher levels. Results from a first experiment using a virtual localization set-up and non-individualized head-related transfer functions (HRTFs) were consistent with this hypothesis, but a second experiment using a free-field set-up showed that vertical localization deteriorates monotonically with increasing level up to $100 \mathrm{~dB}$ SPL. These results suggest that listeners use different cues when localizing sound sources in virtual and free-field conditions. In addition, they confirm that the worsening in vertical localization with increasing level continues beyond $70-80 \mathrm{~dB}$ SPL, the highest levels tested by previous studies. Further, they suggest that vertical localization, unlike high-frequency spectral notch detection, does not rely on an 'ideal observer' analysis of auditory nerve spike trains.

\section{Keywords}

\section{Manuscript region of origin \\ Corresponding Author \\ Corresponding Author's Institution}

Order of Authors

Suggested reviewers
Sound level effect; Head-related transfer function; Vertical localization; Highfrequency spectral cues; Pinna cues; Spectral discrimination

Europe

Frederic Marmel

University of Manchester

Frederic Marmel, Miriam Isabel Marrufo Pérez, Jan Heeren, Stephen Ewert, Enrique Lopez-Poveda

Brad May, John Middlebrooks

\section{Submission Files Included in this PDF}

\section{File Name [File Type]}

CoverLetter_Revision2.docx [Cover Letter]

Rebuttal letter Revision2 Final.docx [Response to Reviewers]

Marmel_etal_HearRes_Highlights_2018.docx [Highlights]

Manuscript_Revision2.docx [Manuscript File]

Figure1.eps [Figure]

Figure2.eps [Figure]

Figure3.eps [Figure]

Figure4.eps [Figure]

To view all the submission files, including those not included in the PDF, click on the manuscript title on your EVISE Homepage, then click 'Download zip file'. 
May 31st 2018

The University of Manchester, M13 9PL, UK

Dear Professor Canlon,

Thank you for the reviews and for giving us the opportunity to revise our manuscript a second time. Please find our revision attached. In preparing our revision, we have carefully considered the comments of the associate editor and of reviewer 2 . We think that the revised manuscript is stronger and hope that it is now suitable for publication.

Sincerely,

Frederic Marmel, on behalf of all authors 


\section{- Comments of the Associate Editor (Brian Moore)}

The two reviewers are largely satisfied with the revised version, although one made some suggestions that you should deal with.

As before, I have marked up a hard copy of the MS with some minor corrections and suggestions.

Line 207: Repeated just below (comment from the marked up hard copy)

This line has been removed.

I did not follow the argument on lines 415-417. Discrimination of the notch center frequency is still involved. And why should an all-information code be used in one task but not the other?

This argument added little to the Discussion and has been removed.

\section{-Reviewer 2}

- The ms has been much improved upon revision. It is nearly ready for publication. The abstract devotes many words to motivating the study and does not summarize the work as well as the Conclusions section. I'd ask the authors to have a look at the abstract.

The abstract has been rewritten to address the reviewer's comment.

Observation: Conclusions are based on the RMS error and the Pearson product moment. These two statistics are not as independent as one might like -- different weightings depending on how compressed the responses are compared to the stimuli.

Somewhere, about line 142 it would be good to know how long a session lasted.

We have included this information.

Line 156: The expected RMS error for random guessing is equal to the separation times sqrt[ $\left.\left(L^{\wedge} 2-1\right) / 6\right]$, which is 55.1 degrees for $L=27$ and a common separation of 5 degrees.

We thank the reviewer for sharing this formula with us, but we have not been able to understand the mathematical reasoning leading to it. Thus, we did not include it in the revised manuscript.

Lines 216 to 252 give a long-winded but not especially useful review of modeling work by some of these authors. The paper would be more readable if this part were shortened.

We believe that this explanation could only be shortened at the expense of clarity. Thus, we have left it unmodified. 


\section{Highlights:}

- Vertical localization deteriorates with increasing sound level up to $100 \mathrm{~dB}$ SPL

- Vertical localization is poor when non-individualized HRTFs are used

- Vertical localization and high-frequency spectral notch detection use different cues

- High-level vertical localization does not use the full auditory nerve information 
1 Effect of sound level on virtual and free-field localization of brief sounds in the anterior

2 median plane

3 Abbreviated title: Effect of level on vertical localization

4 Frederic Marmel $^{\mathrm{a}, \mathrm{b}, 1}$, Miriam I. Marrufo-Pérez ${ }^{\mathrm{a}, \mathrm{b}}$, Jan Heeren ${ }^{\mathrm{d}}$, Stephan Ewert ${ }^{\mathrm{d}}$, Enrique A.

$5 \quad$ Lopez-Poveda ${ }^{\mathrm{a}, \mathrm{b}, \mathrm{c}}$

6

7 a Instituto de Neurociencias de Castilla y León, Universidad de Salamanca, Calle Pintor

$8 \quad$ Fernando Gallego 1, 37007 Salamanca, Spain

$9 \quad{ }^{b}$ Instituto de Investigación Biomédica de Salamanca, Paseo de San Vicente 58-182, 37007

$10 \quad$ Salamanca, Spain

$11{ }^{c}$ Facultad de Medicina, Departamento de Cirugía, Universidad de Salamanca, Avenida Alfonso

$12 \quad X$ “El Sabio” s/n, 37007 Salamanca, Spain

$13{ }^{d}$ Medizinische Physik and Cluster of Excellence Hearing4All, Universität Oldenburg, 26111

$14 \quad$ Oldenburg, Germany

15

$161{ }^{1}$ Corresponding author. E-mail address: frederic.marmel@gmail.com. Present address:

17 Manchester Centre for Audiology and Deafness (ManCAD), Division of Human Communication,

18 Development \& Hearing, School of Health Sciences, Faculty of Biology, Medicine and Health,

19 University of Manchester, Manchester Academic Health Science Centre, Oxford Road, M13

$209 P L, U K$

21

22 Submitted: June, 2018 
Marmel et al. $\quad$ Effect of level on vertical localization

$23 \quad$ Abstract

24 The detection of high-frequency spectral notches has been shown to be worse at 70-80 dB sound 25 pressure level (SPL) than at higher levels up to $100 \mathrm{~dB}$ SPL. The performance improvement at 26 levels higher than 70-80 dB SPL has been related to an 'ideal observer' comparison of 27 population auditory nerve spike trains to stimuli with and without high-frequency spectral 28 notches. Insofar as vertical localization partly relies on information provided by pinna-based 29 high-frequency spectral notches, we hypothesized that localization would be worse at 70-80 dB 30 SPL than at higher levels. Results from a first experiment using a virtual localization set-up and 31 non-individualized head-related transfer functions (HRTFs) were consistent with this hypothesis, 32 but a second experiment using a free-field set-up showed that vertical localization deteriorates 33 monotonically with increasing level up to $100 \mathrm{~dB}$ SPL. These results suggest that listeners use 34 different cues when localizing sound sources in virtual and free-field conditions. In addition, 35 they confirm that the worsening in vertical localization with increasing level continues beyond 36 70-80 dB SPL, the highest levels tested by previous studies. Further, they suggest that vertical 37 localization, unlike high-frequency spectral notch detection, does not rely on an 'ideal observer' 38 analysis of auditory nerve spike trains.

\section{$40 \quad$ Keywords}

41 Sound level effect; Head-related transfer function; Vertical localization; High-frequency spectral 42 cues; Pinna cues; Spectral discrimination 
Marmel et al. $\quad$ Effect of level on vertical localization

\section{Introduction}

45

The ability to localize broadband sounds in the median plane has been shown to deteriorate with increasing sound pressure level (SPL) above about $50 \mathrm{~dB}$ SPL (Hartmann and Rakerd, 1993; Macpherson and Middlebrooks, 2000; Rakerd et al., 1998; Vliegen and Van Opstal, 2004). This level effect is more apparent for brief sounds (a few tens of milliseconds or shorter) than for longer sounds (80 ms or more; Macpherson and Middlebrooks, 2000; Vliegen and Van Opstal, 2004; but see Rakerd et al., 1998 for a level effect with 1-second white noises). Localization in the vertical dimension is thought to rely on high-frequency spectral peaks and notches generated by the filtering action of the pinna (Asano et al., 1990; Iida et al., 2007; Lopez-Poveda and Meddis, 1996; Macpherson and Sabin, 2013; Middlebrooks and Green, 1991). Presumably, auditory peripheral compression, the broadening of cochlear filters, and/or the saturation of auditory nerve fibers' discharge rates make it harder for the peripheral auditory system to resolve pinna cues at high levels (e.g., Rice et al., 1995; Lopez-Poveda, 1996; Macpherson and Middlebrooks, 2000; Macpherson and Sabin, 2013; Reiss et al., 2011; Sachs and Young, 1979). These notions, however, are controversial.

Alves-Pinto and Lopez-Poveda $(2005,2008)$ studied the level dependence of the discriminability of broadband noises with and without a rectangular high-frequency notch centered at $8 \mathrm{kHz}$. They observed that for some participants the ability to detect the highfrequency spectral notch varied non-monotonically with level: just-detectable notch depths were generally smaller for levels below $70 \mathrm{~dB}$ SPL and above $80 \mathrm{~dB}$ SPL than for levels of $70-80 \mathrm{~dB}$ SPL. Insofar as localization in the median plane relies to some extent on high-frequency spectral notches, the work of Alves-Pinto and Lopez-Poveda suggests that vertical localization of brief broadband sounds may not deteriorate monotonically with increasing level. Instead, the level 
67 effect reported previously for levels up to 70-80 dB SPL (Hartmann and Rakerd, 1993;

68 Macpherson and Middlebrooks, 2000; Vliegen and Van Opstal, 2004) could give way to an

69 improvement with increasing level for levels higher than about $80 \mathrm{~dB}$ SPL. The present study

70 investigated this possibility. A first experiment (Section 2) collected elevation judgments in the 71 anterior median plane using virtual sound acoustics for short $(10 \mathrm{~ms})$ broadband noises at sound 72 levels ranging from 30 to $95 \mathrm{~dB}$ SPL. Virtual localization was at chance at 60-80 dB SPL and 73 better than chance at $40-50$ and $90 \mathrm{~dB}$ SPL, which is consistent with the results of Alves-Pinto 74 and Lopez-Poveda (2005). However, a second experiment (Section 3) using loudspeakers placed 75 in the anterior median plane (free-field sound acoustics) showed that localization deteriorated 76 monotonically with increasing level from 50 to $100 \mathrm{~dB}$ SPL for most participants, hence 77 providing evidence against the hypothesis of an improvement for levels higher than about $80 \mathrm{~dB}$ 78 SPL. The results are discussed (Section 4) in terms of the difference between the two tasks and 79 the different mechanisms they may involve.

80

81 2. Experiment 1. Virtual sound localization with non-individualized head-related transfer 82 functions

$83 \quad$ 2.1. Methods

$84 \quad$ 2.1.1. Participants

85 Eleven participants (10 females) were tested, but one was excluded because of an 86 inability to perform the task. The remaining 10 participants were between 22 and 34 years old, 87 with a mean age of 26 years. All of them had audiometric thresholds less than or equal to $20 \mathrm{~dB}$ 88 HL in both ears at octave frequencies spanning $250-8000 \mathrm{~Hz}$ (American National Standards 89 Institute, 2004). Participants were volunteers and were not paid for their service. The human 
experimentation ethical review board of the University of Salamanca approved all procedures.

\subsubsection{Stimuli}

On each trial, the stimulus was a pair of identical 10-ms Gaussian noise bursts separated by a 500-ms silent interval. For each pair, a fresh Gaussian noise was generated in the time domain by drawing pseudorandom sample values from the standard normal distribution (441 samples, corresponding to $10-\mathrm{ms}$ noises generated at a $44100-\mathrm{Hz}$ sampling rate). The sample amplitudes were adjusted to get a root mean square (RMS) level equal to the desired level, and 2ms onset and offset raised-cosine ramps were applied. The resulting noise was duplicated and the two noise segments concatenated with a 500-ms silence between them. Silent 500-ms intervals were added before and after the pair of noises. Out-of-the-head vertical location was simulated by convolving the noises with head-related impulse responses (HRIRs) for a KEMAR (Knowles Electronics model DB-4004 with the "small” pinna model DB-061), as measured by Gardner and Martin (1995). The "full” HRIRs (containing the response of the measurement system and the KEMAR ear canal resonance) were used rather than the equalized "diffuse" versions, because localization seemed easier to the first two authors this way. Fourteen virtual vertical sources were simulated in the anterior median plane, with elevation angles spanning $-40^{\circ}$ to $90^{\circ}$ in $10^{\circ}$ steps. Figure 1 shows the frequency spectra of the HRIR for each elevation angle, hereinafter referred to as head-related transfer functions (HRTFs). The noises had levels of 30, 40, 50, 60, 70, 80, 90, and $95 \mathrm{~dB}$ SPL.

\subsubsection{Apparatus}

All stimuli were generated digitally using custom Matlab software (The Mathworks, 
Natick, Massachusetts, USA). Stimuli were digital-to-analog converted using an RME Fireface

\subsubsection{Procedure}

The experimental procedure was controlled via custom Matlab software. Each participant completed four sessions of eight blocks (corresponding to the eight target levels). Each session started with the 60-dB-SPL block followed by the 50-, 40-, 30-, 70-, 80-, 90-, and 95-dB-SPL blocks, in that order. Within each block, 14 stimuli corresponding to the 14 input angles were presented randomly. The computer screen displayed a picture of a head in a circle with 27 stimulus presentation, participants had to judge the elevation of the sound by clicking on one of the buttons. The participant's response (no time limit) triggered the presentation of the next stimulus. No feedback was given.

Before beginning the experiment, participants went through a training phase: the task was explained to them and they were requested to train on a demonstration set-up. The demonstration 
set-up showed the same display as the experimental set-up except that there were only 14 buttons

\subsubsection{Statistics}

For each participant, two measures of virtual localization accuracy were calculated for each session and target level: the Pearson correlation coefficient between the actual and response angles, and the RMS angle error defined as the RMS value of the differences between the actual and the response angles (e.g., Middlebrooks, 1999; Majdak et al., 2013). These two measures were analyzed with repeated-measures ANOVAs using session (last three sessions) and sound level (the eight target levels) as factors. Bonferroni-corrected t-tests were then used to compare accuracy to chance (for each target level), as well as to compare pairs of conditions. As a measure of effect size, the Pearson correlation coefficients ( $r$ values) were calculated from the $t$ values and the degrees of freedom (df) with the formula $r=\sqrt{\frac{t^{2}}{t^{2}+d f}}$ (Field, 2005, p.294; Rosenthal, 1991, p.19). Monte-Carlo simulations in Matlab were run to estimate chance performance for RMS errors: random guessing would lead to an RMS error of $55.4^{\circ}$ (average of 
100000 simulations, standard deviation $=8.3^{\circ}$ ). Chance performance for correlation coefficients

is 0 . All statistical analyses were performed using the computing environment $\mathrm{R}$ ( $\mathrm{R}$ Core Team, $1612012)$

\subsection{Results}

Both the RMS errors and the correlation coefficients showed an effect of sound level [RMS errors: $F(7,63)=2.37 ; p<0.05$, correlation coefficients: $F(7,63)=2.90 ; p<0.05$ ]. Neither the RMS errors nor the correlation coefficients showed an effect of experimental session. Thus, results for the three sessions were averaged for further analyses. Figure 2 plots the resulting RMS errors (triangles, left y-axis) and correlation coefficients (circles, right y-axis) for each participant and for the group mean. For RMS errors, seven out of the ten participants (the exceptions being SA1, SA5 and SA8) had a local mid-to-high-level peak (defined as a level between 60 and $80 \mathrm{~dB}$ SPL with an RMS error higher than at the two lower and at the two higher neighboring levels), indicating worse performance at mid-to-high levels than at lower and higher levels. For the correlation coefficients, all participants except SA9 showed a local mid-to-highlevel trough (defined as a level between 60 and $80 \mathrm{~dB}$ SPL with a correlation coefficient lower than at the two lower and at the two higher neighboring levels), again indicating worse performance at mid-to-high levels than at lower and higher levels. For participant SA9, a local trough was observed at $50 \mathrm{~dB}$ SPL.

Turning to group results, the comparisons to chance performance for RMS errors (onetailed one-sample t-tests, Bonferroni-adjusted $p=0.00625$ ) showed RMS errors better (smaller) than chance at $40 \mathrm{~dB}$ SPL $[t(9)=-5.88 ; p=0.0001 ; 95 \%$ confidence interval for the difference $181(\mathrm{CI})=[-12.3-5.46] ; r=0.89]$, at $50 \mathrm{~dB} \operatorname{SPL}[t(9)=-3.65 ; p=0.0027 ; \mathrm{CI}=[-10.9-2.57] ; r=$ 
$0.77]$, and at $90 \mathrm{~dB}$ SPL $[t(9)=-5.21 ; p=0.0003 ; \mathrm{CI}=[-8.78-3.46] ; r=0.87]$. The three betterthan-chance conditions (40,50, and $90 \mathrm{~dB}$ SPL) were compared with each of the five other conditions $(30,60,70,80$, and $95 \mathrm{~dB}$ SPL), resulting in fifteen paired t-tests and a Bonferroniadjusted $p$ of 0.0033 (one-tailed). RMS errors were smaller at 40 and $90 \mathrm{~dB}$ SPL than at $70 \mathrm{~dB}$ SPL [for $40 v s .70 \mathrm{~dB}: t(9)=-3.84 ; p=0.0020 ; \mathrm{CI}=[-13.5-3.49] ; r=0.79]$; for $90 v s .70 \mathrm{~dB}$ : $t(9)=-4.17 ; p=0.0012 ; \mathrm{CI}=[-8.84-2.62] ; r=0.81]$.

For the correlation coefficients between response and target angles, the comparisons to chance performance (one-tailed one-sample t-tests, Bonferroni-adjusted $p=0.00625$ ) showed coefficients better (higher) than chance at $40 \mathrm{~dB}$ SPL $[t(9)=4.63 ; p=0.0005 ; \mathrm{CI}=[0.130 .39] ; r$ $=0.84]$, at $50 \mathrm{~dB}$ SPL $[t(9)=3.17 ; p=0.0055 ; \mathrm{CI}=[0.050 .33] ; r=0.73]$, and at $90 \mathrm{~dB} \mathrm{SPL}$ $[t(9)=5.95 ; p=0.0001 ; \mathrm{CI}=[0.110 .25] ; r=0.89]$. The same fifteen pairwise comparisons as above (one-tailed paired t-tests, Bonferroni-adjusted $p=0.0033$ ) showed correlation coefficients higher at 40 than at $70 \mathrm{~dB}$ SPL $[t(9)=3.91 ; p=0.0018 ; \mathrm{CI}=[0.130 .47] ; r=0.79]$, as well as correlation coefficients higher at 90 than at 70 and $80 \mathrm{~dB}$ SPL [for $90 v s .70 \mathrm{~dB}: t(9)=3.87 ; p=$ 0.0019; $\mathrm{CI}=[0.09$ 0.35]; $r=0.79$; for 90 vs. $80 \mathrm{~dB}: t(9)=4.97 ; p=0.0004 ; \mathrm{CI}=[0.090 .24] ; r=$ $0.86]$.

In summary, the results indicated that virtual localization accuracy varied nonmonotonically with sound level. Performance was poor overall, but was significantly better than chance at 40, 50, and $90 \mathrm{~dB}$ SPL. Note that all the t-tests indicated large effects, as all effect sizes ( $r$ values) were well above 0.5 , the threshold value for large effects (Cohen, 1992). Between 40 and $90 \mathrm{~dB}$ SPL, performance deteriorated with increasing level up to $70 \mathrm{~dB}$ SPL and then improved as level increased to $90 \mathrm{~dB}$ SPL (bottom-right panel of Figure 2). Importantly, performance at $60 \mathrm{~dB}$ SPL was at chance in spite of the training phase being carried at this level, 
while performance at 40,50 and $90 \mathrm{~dB}$ SPL was better than chance in spite of participants not being trained at these levels. At-chance performance at $30 \mathrm{~dB}$ SPL may be explained by the decline in vertical-localization performance for sensation levels below $\sim 30 \mathrm{~dB}$, which is hypothesized to reflect the high-frequency portion of the stimulus falling below absolute

\section{9 threshold (Sabin et al., 2005).}

\subsection{Discussion}

Experiment 1 showed virtual localization accuracy to be worse at mid-to-high levels than at lower or higher levels. This is similar to the level effect reported for high-frequency spectral notch detection by Alves-Pinto and Lopez-Poveda $(2005,2008)$, as can be seen by visually comparing the RMS errors in Figure 2 to their notch depth thresholds (see Fig. 4 in Alves-Pinto and Lopez-Poveda, 2005; and Fig. 2 in Alves-Pinto and Lopez-Poveda, 2008). The pattern of mean RMS errors across sound levels also looked similar to the threshold spectral notch depths predicted in Fig. 8 of Alves-Pinto et al. (2014) when performing ideal-observer analyses on either the responses of low-spontaneous rate (LSR) auditory nerve fibers or on the responses of a combination of LSR and medium-spontaneous rate (MSR) fibers to noises with and without high-frequency spectral notches. When the ideal-observer analysis was applied to the responses of high-spontaneous rate (HSR) fibers, however, the predicted thresholds increased monotonically up to $90-100 \mathrm{~dB}$ SPL. The comparison of the results of the present Experiment 1 to the spectral notch detection studies suggests that LSR and MSR fibers may be more important for virtual localization in the anterior median plane than HSR fibers, for low as well as for high sound levels, and that the level effect on virtual localization may be predicted from responses of LSR and MSR fibers to high-frequency spectral notches. 
Lopez-Poveda et al. (2008) proposed an explanation for the non-monotonic level effect observed in the spectral notch detection studies. They used a computational model of the auditory periphery to simulate the effect of level on two different representations of highfrequency spectral notches in the receptor potential of the population of inner hair cells (IHCs). One representation involved plotting the RMS receptor potential for each IHC as a function of the IHC characteristic frequency. The mean auditory nerve discharge rate is proportional to the average IHC receptor potential (e.g., Cheatham and Dallos, 2001). Therefore, this representation may be thought of as related to an auditory nerve rate profile. The other representation involved calculating the fast Fourier transform (FFT) of the receptor potential for each IHC and adding the resulting amplitude spectra across IHCs in the frequency domain (phase disregarded). The FFT of the receptor potential reflects the low-pass filtering characteristics of the IHC, which determine the phase locking of auditory nerve discharges (e.g., Palmer and Russell, 1986). Hence, this latter representation may be thought of as related to the timing of spikes for the population of auditory nerve fibers (ANF). The former ('rate profile') representation was less and less sensitive to spectral notches as level increased up to $100 \mathrm{~dB}$ SPL, whereas the latter ('spike timing') representation was less and less sensitive with increasing level up to 60-70 $\mathrm{dB}$ SPL but was more and more sensitive to spectral notches as level increased further, up to $100 \mathrm{~dB}$ SPL. This suggested that the non-monotonic level effect found in the spectral notch detection studies might be related to differences in the timing of ANFs spikes and not to differences in the average spike rates (the rate profile) (for further details, see Lopez-Poveda et al., 2008).

The consistency between the level effect found in Experiment 1 and the level effect found in high-frequency notch detection studies (Alves-Pinto and Lopez-Poveda, 2005, 2008; AlvesPinto et al., 2014; Lopez-Poveda et al., 2008) supported both the non-monotonic effect of level 
on high-frequency spectral notch detection and the importance of high-frequency cues for virtual vertical localization. However, a study with real sources (or individualized HRTFs) was still needed to confirm the non-monotonic effect of level for 'real' vertical localization. Non-

\section{Experiment 2. Free-field localization}

\subsection{Methods}

\subsubsection{Participants}

Ten participants (4 females), different from those who participated in Experiment 1, were tested. Their ages ranged from 15 to 30 years, with a mean age of 26 years. Nine of them had prior experience in hearing experiments. All of them had audiometric thresholds less than or equal to $20 \mathrm{~dB} \mathrm{HL}$ in both ears at octave frequencies spanning 250-8000 Hz (American National Standards Institute, 2004), except for one participant who had a threshold of $25 \mathrm{~dB}$ HL at 8000 $\mathrm{Hz}$ for both ears. Participants were volunteers and were not paid for their service. The human experimentation ethical review board of the University of Oldenburg approved all procedures.

\subsubsection{Stimuli}

Pairs of noise bursts were generated as in Experiment 1. The noise levels were 30, 40, 50, $60,70,80,90,95$, and $100 \mathrm{~dB}$ SPL. 


\subsubsection{Apparatus}

The loudspeaker set-up for Experiment 2 consisted of eleven Genelec 8030B studio

\subsubsection{Procedure}

The experimental procedure was controlled via custom Matlab software. Unlike in Experiment 1, the experimenter controlled the software. Upon each stimulus presentation, participants had to judge orally from which of the loudspeakers the stimulus was played. The experimenter heard the response via a talkback connection and entered it into the measurement 
software using the same graphical user interface (GUI) as in Experiment 1. Entering the

participant's response (no time limit) to the GUI triggered the presentation of the next stimulus. No feedback was given. Each participant completed four sessions of nine blocks (corresponding to the nine target levels). Each session started with the 60-dB-SPL block, followed by the 50-, 40-, 30-, 70-, 80-, 90-, 95-, and 100-dB-SPL blocks in that order. Within each block, eleven stimuli corresponding to the eleven angles were presented randomly. Before the first session, the experimenter gave a demonstration of the stimuli from each direction. Loudspeakers/directions were demonstrated in the order of the loudspeaker labels with prior announcement of the number of the next loudspeaker via the talkback system. Participants completed all the testing within one day.

\subsubsection{Statistics}

Unlike Experiment 1, performance was good in the first of the four experimental sessions. Thus, the first session was not regarded as training and the results for all four sessions were used for statistical analyses. For each of the ten participants, two measures of localization accuracy were calculated for each target level: the Pearson correlation coefficient between the actual and the response angles, and the RMS value of the localization error. These two measures were not normally distributed for all sound levels. Therefore, they were analyzed with the aligned-ranked statistical technique for nonparametric factorial ANOVAs proposed by Wobbrock et al. (2011) (using the ARTool package in R), with sound level and session as factors. Significant effects were investigated further with post hoc pairwise comparisons, in which $p$-values were Tukey-corrected for multiple comparisons. All statistical analyses were performed using the computing environment R (R Core Team, 2012). 
Monte-Carlo simulations in Matlab were run to estimate chance performance for RMS errors: random guessing would lead to an RMS error of $44.1^{\circ}$ (average of 100000 simulations, standard deviation $=7 \cdot 6^{\circ}$ ). Chance performance for correlation coefficients is 0 .

\subsection{Results}

Figure 3 plots the RMS errors and the correlation coefficients for each session and sound level, averaged over the 10 participants. Both measures showed an effect of sound level [RMS errors: $F(8,315)=154 ; p<0.001$, correlation coefficients: $F(8,315)=150 ; p<0.001]$ and, unlike Experiment 1, an effect of session [RMS errors: $F(3,315)=7.25 ; p<0.001$, correlation coefficients: $F(8,315)=14.6 ; p<0.001]$. The correlation coefficients showed an interaction between sound level and session $[F(24,315)=2.30 ; p<0.001]$. Post-hoc comparisons showed that performance was better in session \#2 than in sessions \#1, \#3 and \#4 [session \#4: $p<0.001$ for both RMS errors and correlation coefficients; session \#3: $p<0.05$ for RMS errors and $p<$ 0.001 for correlation coefficients; session $\# 1: p<0.01$ for correlation coefficients only]. The better performance in session \#2 was most apparent at $100 \mathrm{~dB}$ SPL. Performance improved with sound level increasing from $30 \mathrm{~dB}$ to $40-50 \mathrm{~dB}$ SPL and then worsened monotonically up to 100 dB SPL (except for session \#2).

Figure 4 plots the RMS errors and the correlation coefficients averaged over the four sessions as a function of sound level for each participant individually and for the 10 participants averaged. Figure 4 also plots the chance values for RMS errors and correlation coefficients. Several participants showed a performance improvement from $30 \mathrm{~dB}$ to $40-50 \mathrm{~dB}$ SPL and all participants showed a performance worsening from $60 \mathrm{~dB}$ to $90 \mathrm{~dB}$ SPL. For levels between 90 $\mathrm{dB}$ and $100 \mathrm{~dB}$ SPL, performance worsened further for six participants (OL1 and OL4 to OL8). 


\subsection{Discussion}

Performance was better in Experiment 2 than in Experiment 1, which highlights the difficulty of the virtual localization task in Experiment 1 and illustrates how damaging the use of non-individualized HRTFs and the lack of visual cues was to performance. It is surprising that the use of free-field localization not only changed localization accuracy but also the dependence of localization accuracy on sound level. Contrary to Experiment 1, the participants in Experiment 2 did not show better performance at very high levels than at mid-to-high levels. Thus, Experiment 2 failed to support the hypothesis of a vertical localization improvement at very high levels. The methodological differences between the two experiments and the variability between participants in Experiment 2 suggest that different types of cues may have been used to perform the two tasks of the present study, and that these cues changed in a different way with sound level.

\section{General discussion}

\subsection{Comparison to vertical localization performance in previous studies}

Virtual localization performance in Experiment 1 was much poorer than free-field localization performance in Experiment 2. The grand average RMS error (across all participants, conditions, and experimental sessions) was $51^{\circ}$ in Experiment 1 and $20^{\circ}$ in Experiment 2, and the grand average correlation coefficient was 0.13 in Experiment 1 and 0.74 in Experiment 2. The use of virtual acoustics is known to impair performance, notably by increasing the number of front-back and up-down confusions (e.g., Wenzel et al., 1993). The difference between Experiments 1 and 2 is consistent with results from Middlebrooks (1999), who compared vertical 
localization performance using individualized (own) and non-individualized (others) HRTFs and observed an increase of RMS errors from $\sim 28^{\circ}$ in the former condition to $\sim 42^{\circ}$ in the latter. The

\subsection{Level-dependent discriminability of HRTFs}

The worsening of free-field localization with increasing sound level (Experiment 2,

Figures 3 and 4) is consistent with the notions that (1) localization in the vertical plane relies on a rate profile representation of high-frequency spectral notches in the auditory nerve; and (2) the quality of that representation decreases with increasing sound level above about $40 \mathrm{~dB}$ SPL (e.g., Rose et al., 1971; Rice et al., 1995; Lopez-Poveda, 1996; Alves-Pinto and Lopez-Poveda, 2008; Lopez-Poveda et al., 2008; Alves-Pinto et al., 2014). This worsening, however, contrasts with the non-monotonic dependence on level observed for high-frequency spectral notch detection (Alves-Pinto and Lopez-Poveda, 2005, 2008) and with information for high-frequency spectral notch detection being encoded in the timing of auditory nerve discharges (Alves-Pinto et al., 2014; Lopez-Poveda et al., 2008). Taken together, this evidence suggests that high-frequency spectral notch detection and vertical localization probably rely on different cues and/or neural 388 codes. 
The notch detection task of Alves-Pinto and Lopez-Poveda $(2005,2008)$ and the simulations of Alves-Pinto et al. (2014) involved discriminating between noises with and without a high-frequency spectral notch. Vertical localization, by contrast, possibly requires being able to discriminate location-dependent spectral shapes generated by the filtering action of the pinnae (Middlebrooks and Green, 1991). Specifically, vertical localization might depend on the ability to discriminate the center frequency of narrow, elevation-dependent high-frequency spectral notches (e.g., Hebrank and Wright, 1974; Butler and Belendiuk, 1977; Watkins, 1978; Bloom, 1977) rather than on the ability to detect those notches (i.e., to discriminate between notch and no-notch stimuli). Perhaps notch frequency discrimination worsens monotonically with increasing level while notch depth detection varies non-monotonically with increasing level. This might explain the different level dependence for the spectral notch detection studies and for Experiment 2.

Experiment 2 was a 'true' localization experiment, and as such probably relied on a comparison between the auditory nerve rate profile generated by the stimulus with a "learnt and memorized" rate profile. On the other hand, the mismatch between the non-individualized HRTFs of Experiment 1 and participants' own HRTFs would have made such a comparison strategy inefficient, which might have led participants in Experiment 1 to focus more on the differences between stimuli. Notch detection possibly relies on an "ideal observer" (or allinformation) type of comparison between auditory nerve spike trains generated by the notch and no-notch stimuli. Participants tapping into an all-information neural code, as in Alves-Pinto et al. (2014), might explain the better-than-chance performance at 90 dB SPL in Experiment 1. 


\section{Conclusions}

The present study investigated the apparent discrepancy between vertical localization (elevation perception) getting harder at higher sound levels and high-frequency spectral notch detection getting easier at sound levels above 70-80 dB SPL. Contrary to our hypothesis, this discrepancy cannot be explained by the sound levels in previous vertical localization studies being insufficiently high. Instead, the present results confirmed that vertical localization deteriorates with increasing level up to $100 \mathrm{~dB}$ SPL. The performance improvement observed for levels higher than 70-80 dB SPL in notch detection studies and in Experiment 1 is likely to be related to a discrimination cue not used for vertical localization. This cue might arise from an 'all-information' neural representation of the stimuli that is not relevant to vertical localization.

\section{Acknowledgments}

This work was supported by the Spanish Ministry of Economy and Competitiveness (BFU2012-39544-C02 and BFU2015-65376-P), European Regional Development Funds, the University of Salamanca and Banco Santander. 


\section{References}

Alves-Pinto A, Lopez-Poveda EA. Detection of high-frequency spectral notches as a function of level. J Acoust Soc Am. 2005; 118:2458-69.

Alves-Pinto A, Lopez-Poveda EA. Psychophysical assessment of the level-dependent representation of high-frequency spectral notches in the peripheral auditory system. $\mathrm{J}$ Acoust Soc Am. 2008; 124:409-21.

Alves-Pinto A, Palmer AR, Lopez-Poveda EA. Perception and coding of high-frequency spectral notches: potential implications for sound localization. Front Neurosci. 2014; 8. doi: 10.3389/fnins.2014.00112.

American National Standards Institute. Methods for manual pure tone threshold audiometry (ANSI/ASA S3.21-2004). New York: American National Standards Institute; 2004.

Asano F, Suzuki Y, Sone T. Role of spectral cues in median plane localization. J Acoust Soc Am. 1990; 88:159-68.

Bloom PJ. Determination of monaural sensitivity changes due to the pinna by use of minimumaudible-field measurements in the lateral vertical plane. J. Acoust. Soc. Am. 1977; 61:8208.

Butler RA, Belendiuk K. Spectral cues utilized in the localization of sound in the median sagittal plane. J. Acoust. Soc. Am. 1977; 61:1264-9.

Cheatham MA, Dallos P. Inner hair cell response patterns: Implications for low-frequency hearing. J. Acoust. Soc. Am. 2001; 110:2034-2044.

Cohen J. A power primer. Psychol Bull. 1992; 112:155-9.

Field A. Discovering statistics using SPSS. 2 ${ }^{\text {nd }}$ ed. London: Sage; 2005. p. 294.

Gardner WG, Martin KD. HRTF measurements of a KEMAR. J Acoust Soc Am. 1995; 97:3907-8.

Hebrank J, Wright D. Spectral cues used in the localization of sound sources on the median plane. J. Acoust. Soc. Am. 1974; 56:1829-34.

Hartmann WM, Rakerd B. Auditory spectral discrimination and the localization of clicks in the sagittal plane. J Acoust Soc Am. 1993; 94:2083-92.

Iida K, Itoh M, Itagaki A, Morimoto M. Median plane localization using a parametric model of the head-related transfer function based on spectral cues. Appl Acoust 2007; 68:835-850.

Lopez-Poveda EA. The physical origin and physiological coding of pinna-based spectral cues 
[PhD Thesis]. Loughborough (UK): Loughborough University; 1996.

Lopez-Poveda EA, Meddis R. A physical model of sound diffraction and reflections in the human concha. J Acoust Soc Am. 1996; 100:3248-59.

Lopez-Poveda EA, Alves-Pinto A, Palmer AR, Eustaquio-Martin A. Rate versus time representation of high-frequency spectral notches in the peripheral auditory system: a computational modeling study. Neurocomputing 2008; 71:693-703.

Majdak P, Masiero B, Fels J. Sound localization in individualized and non-individualized crosstalk cancellation systems. J Acoust Soc Am. 2013; 133:2055-68.

Macpherson EA, Middlebrooks JC. Localization of brief sounds: Effects of level and background noise. J Acoust Soc Am. 2000; 108:1834-49.

Macpherson EA, Sabin AT. Vertical-plane sound localization with distorted spectral cues. Hearing Res. 2013; 306:76-92.

Middlebrooks JC, Green DM. Sound localization by human listeners. Annu. Rev. Psychol. 1991; 42:135-139.

Middlebrooks JC. Virtual localization improved by scaling nonindividualized external-ear transfer functions in frequency. J Acoust Soc Am. 1999; 106:1493-1510.

Palmer AR, Russell IJ. Phase-locking in the cochlear nerve of the guinea-pig and its relation to the receptor potential of inner-hair cells. Hearing Res. 1986; 24:1-15.

R Core Team. R: A Language and Environment for Statistical Computing. R Foundation for Statistical Computing. Vienna, Austria; 2012. ISBN: 3-900051-07-0. Available: http://www.R-project.org.

Rakerd B, Vander-Velde TJ, Hartmann WM. Sound localization in the median sagittal plane by listeners with presbyacusis. J Am Acad Audiol. 1998; 9:466-79.

Reiss LAJ, Ramachandran R, May BJ. Effects of signal level and background noise on spectral representations in the auditory nerve of the domestic cat. J Assoc Res Otolaryngol. 2011; $12: 71-88$.

Rice JJ, Young ED, Spirou GA. Auditory-nerve encoding of pinna-based spectral cues: Rate representation of high-frequency stimuli. J. Acoust. Soc. Am. 1995; 97:1764-1766.

Rose JE, Gross NB, Geisler CD, Hind JE. Some effects of stimulus intensity on response of auditory-nerve fibers in the squirrel monkey. J Neurophysiol. 1971; 34:685-699.

Rosenthal R. Meta-analytic procedures for social research. Newbury Park (CA): Sage; 1991. 
p.19.

Sabin AT, Macpherson EA, Middlebrooks JC. Human sound localization at near-threshold levels. Hearing Res. 2005; 199:124-34.

Sachs M, Young E. Encoding of steady state vowels in the auditory nerve: representation in terms of discharge rate. J Acoust Soc Am. 1979; 66:470-9.

Vliegen J, Van Opstal AJ. The influence of duration and level on human sound localization. J Acoust Soc Am. 2004; 115:1705-13.

Watkins AJ. Psychoacoustical aspects of synthesized vertical locale cues. J. Acoust. Soc. Am. 1978; 63:1152-65.

Wenzel EM, Arruda M, Kistler DJ, Wightman FL. Localization using nonindividualized head-related transfer functions. J Acoust Soc Am. 1993; 94:111-23.

Wobbrock JO, Findlater L, Gergle D, Higgins JJ. The Aligned Rank transform for nonparametric factorial analyses using only ANOVA procedures. Paper presented at the ACM Conference on Human Factors in Computing Systems (CHI '11). 2011, May 7-12: Vancouver, British Columbia. 


\section{FIGURE CAPTIONS}

\section{FIG. 2. Virtual localization performance in Experiment 1: individual and group mean}

performance. Angular RMS errors (triangles, left y-axis) and correlation coefficients between actual angles and response angles (circles, right y-axis), were averaged for each participant and for the group mean over the last three experimental sessions, and are plotted as a function of sound level. For the group mean, error bars depict \pm 1 standard error of the mean. The horizontal dashed and dotted lines depict chance performance for the RMS errors and correlation coefficients, respectively. For the group mean, downward and upward pointing arrows indicate sound levels at which mean RMS errors and correlation coefficients were significantly better than the values at chance.

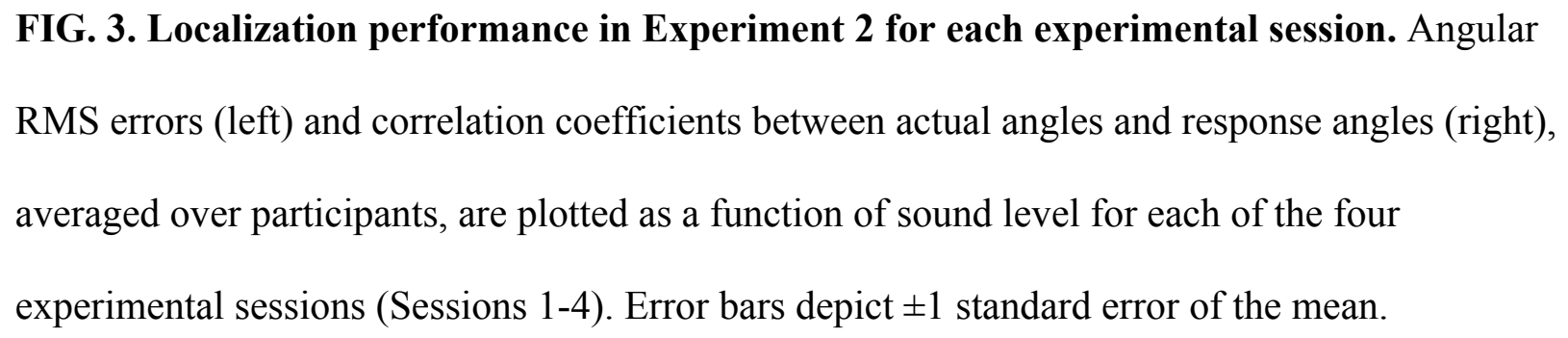

\section{FIG. 4. Localization performance in Experiment 2: individual and group mean performance. As Figure 2, but for Experiment 2.}




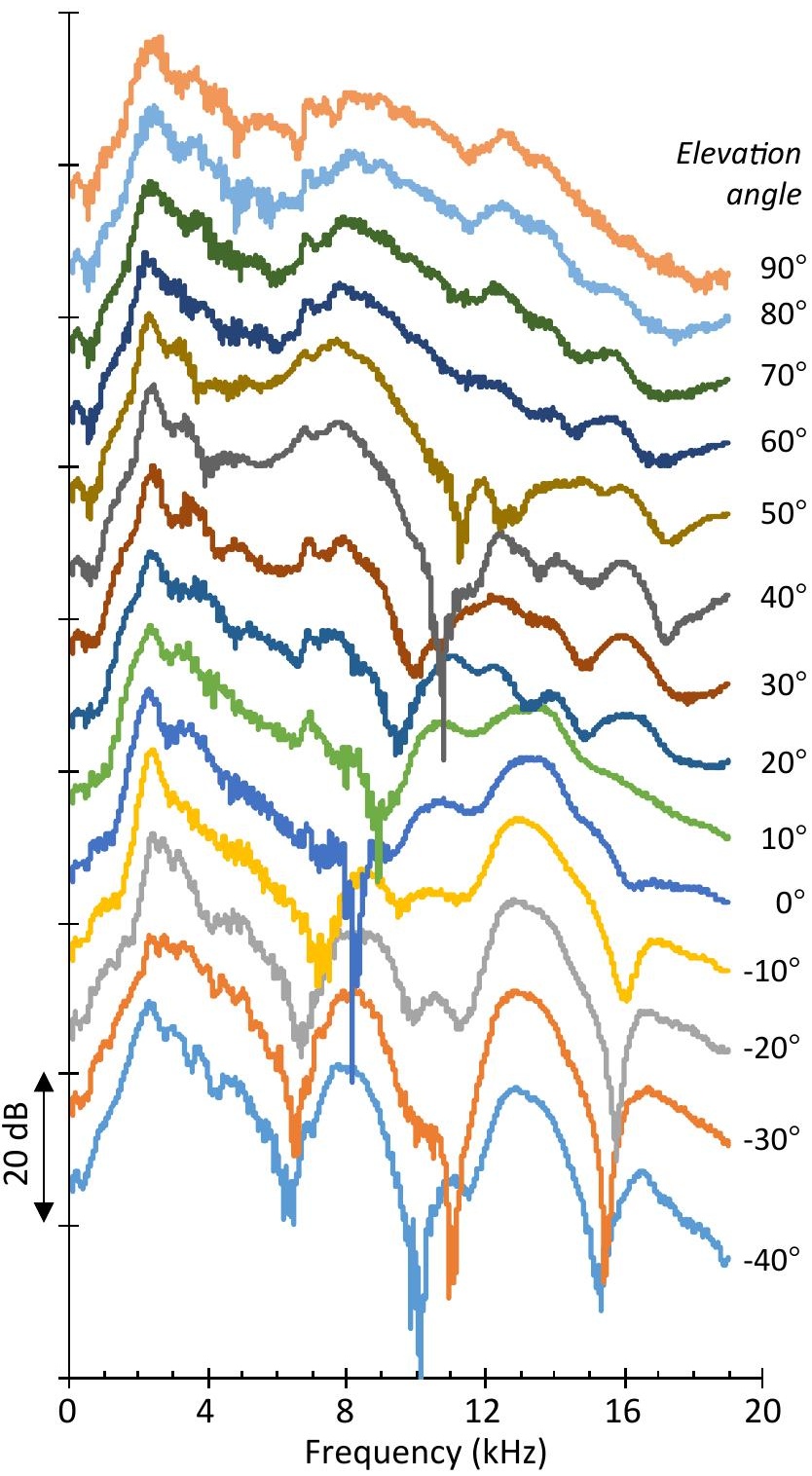




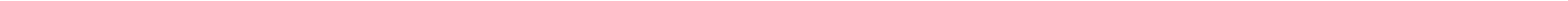




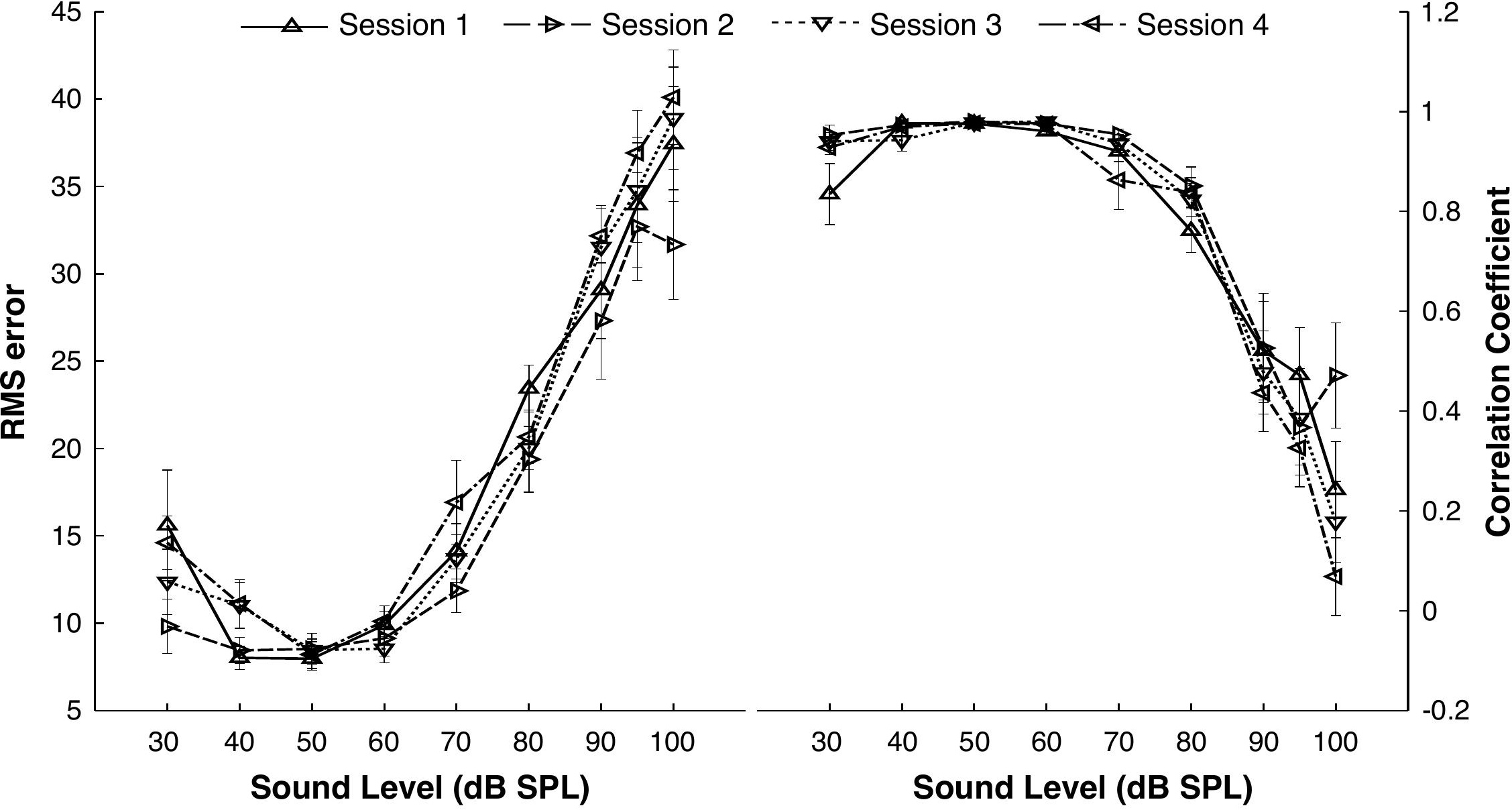




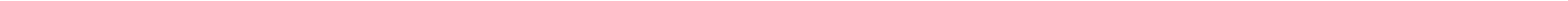

